\section{NEW TREATMENT FOR CLEFT PALATES}

Researchers from the UK and USA have invented a hydrogel material that promises better treatment for cleft palates. The preliminary results on the hydrogel, which has been studied using the Science and Technology Facilities Council's ISIS neutron source, show treatment for severe cleft palates could be carried out without the need for complex surgery.

Cleft palates are currently repaired by surgically repositioning the available palatal mucosa in order to cover the gap in the palate. However, if the cleft defect is too wide there may be insufficient local tissue available to close the gap without undertaking quite radical surgery. It is these severe cases that can cause future complications for infants as they develop into adults - particularly with speech and facial growth problems.

The team of researchers at the University of Oxford, the John Radcliffe Hospital in Oxfordshire and the Georgia Institute of Technology in the United States says the new potential treatment for severe cases involves inserting a small plate made of an anisotropic hydrogel material (similar to that used in contact lenses) under the mucosa of the roof of the mouth of the patient.

The hydrogel gradually expands as fluid is absorbed, encouraging skin growth over and around the plate in 2011. a process known as 'tissue expansion'. When sufficient skin has been generated to repair the palatal cleft, the plate is removed and the cleft is repaired by using this additional tissue. The success of the preliminary results of selfinflating anisotropic hydrogel tissue expanders mean clinical trials in this area are expected to take place early

'Babies born with cleft palates usually have problems feeding, and may have speech difficulties in later life, as well as issues with their hearing, dentition and facial growth,' says $\mathrm{Mr}$ Marc Swan, a plastic surgeon at the

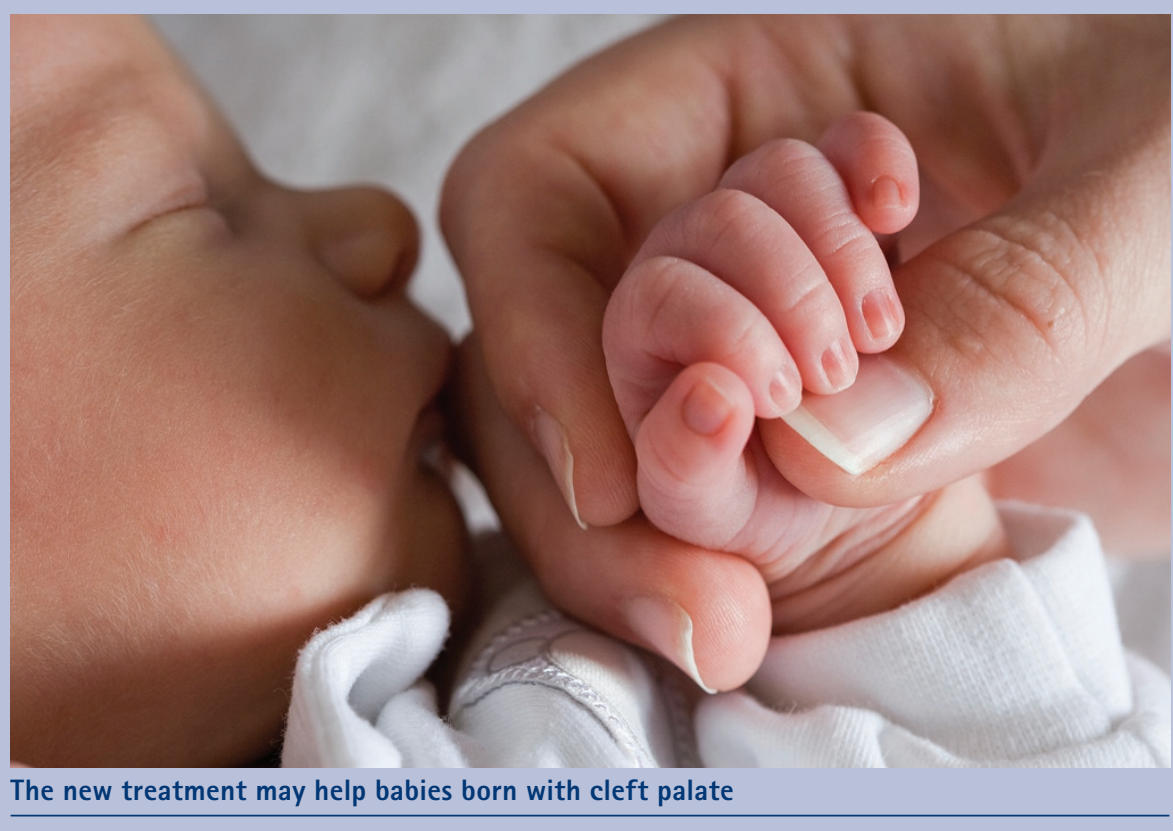

John Radcliffe Hospital in Oxford, and the instigator of the study. 'The severest cases often have the least favourable outcomes and unfortunately these are the most challenging children to treat surgically.'

Rosanna Preston, CEO of CLAPA (The Cleft Lip and Palate Association), commenting on the research said, 'Facial clefts of the lip or palate are the most common birth defect and it is vital that we continue to explore new treatments to help those affected. This research is particularly interesting as it addresses the most severe cases where the effects on the child's development may be greatest. We will be excited to see the results of the clinical trials.'

\section{PILOTS UNDERWAY}

At least 30 NHS dental practices will shortly be trialling new ways to improve services for patients with improved access and new ways of measuring quality as they become pilot sites for the recommendations of the Steele Review of NHS dentistry.

Nearly 30 sites around the country will now pilot new ways of improving services for patients and the NHS from April.

One of the successful sites is City and Hackney where the new blended contract is being trialled, which sees dentists being directly rewarded for the number of patients seen, the level of treatment each patient receives and the quality of that care.

Professor Jimmy Steele, who led the Independent Review of NHS Dental Services, made over 30 recommendations to help improve oral health, increase access and ensure high quality dental care for patients in his final report published in June last year.

Different methods of delivering these recommendations will be piloted thoroughly over the next two years to ensure they meet the needs of the NHS and patients, but the flexibility of the current dental contract means that if the local NHS wants to adopt changes sooner they are able to.
The British Dental Association (BDA) reaffirmed its support for the pilots. Dr John Milne, Chair of the BDA's General Dental Practice Committee, said, 'The BDA has called for reform to deliver an NHS dental system that works better for patients and dentists alike, and we support the process of piloting the conclusions of Professor Steele's review. The two keys to the success of these pilots are meaningful engagement with the profession and proper evaluation of their results. Engagement so far has been good and must continue. These pilots must now be given the time they need to properly assess the proposed changes and allow reflection on the results they produce.' 